\title{
Zwischen Gefährdung und Gefährlichkeit. Wechselbeziehungen sicherheitsbezogener Zugehörigkeitsbestimmungen und Verantwortungshierarchien in den 1970er Jahren
}

\author{
Christine G. Krüger
}

\section{Abstract}

Using the squatter movement and the construction of large-scale housing estates of the 1970s as examples, this contribution examines the interplay between two pairs of categories of difference that are rooted in perceptions of security: 'endangered'/ 'dangerous' and 'in need of protection'/ 'entrusted with the task of protection'. Both, so this contribution illustrates, play a constitutive part in the construction of group identities and do so in different ways. Whereas the distinction between endangered and dangerous is often a factor in determining group affiliation, the distribution of responsibility in matters of protection, the second category of difference, governs hierarchies within a collective. Both pairs of categories of difference are closely related and easily give way to the other, and both usually overlap with other categories of difference that derive from, for instance, social background, age or gender. On this basis, one can explain how the family squatters in London, who sought to present themselves to the public as being in need of protection, were actually perceived by many contemporaries as being dangerous dropouts or that it would have taken no more than a small step to turn the endangered children of the large housing estates into dangerous hooligans in the public eye.

Sicherheitsziele sind oftmals der Ausgangspunkt für Gruppenbildungsprozesse und die damit verknüptte Entstehung von Differenzkategorien (vgl. Conze 2017, S. 150-159). Individuen schließen sich zusammen, um wahrgenommene Bedrohungen effektiver abzuwehren. Nicht selten geht dies mit einer territorialen Abgrenzung einher, etwa dem Bau einer Stadtmauer oder der Errichtung von Grenzpfählen, die einen Sicherheitsraum absteckt und gleichzeitig die Zugehörigkeit zu einem Kollektiv regelt. Hand in Hand damit gehen kommunikative In- und Exklusionsprozesse.

Mit der Unterscheidung zwischen gefährlich und ungefährlich entsteht aber nur eines der Differenzkategorienpaare, die mit dem Verweis auf Sicherheit definiert werden: Darüber hinaus legen Sicherheitsentwürfe gesellschaftliche Verantwortungsbereiche fest und differenzieren zwischen 
schutzbedürftig und schutzbeauftragt. Für beide Arten der Zuordnung gilt, dass sie sich in der Regel mit anders bestimmten Differenzkategorien wie etwa Nationalität, Klasse, Geschlecht oder Generation überlagern. Die Dynamik der daraus entstehenden Wechselbeziehungen soll im Folgenden an zwei Fallbeispielen aus den 1970er Jahren untersucht werden.

Die 1970er Jahre gelten in der historischen Forschung als Phase einer starken gesellschaftlichen Verunsicherung (vgl. Geyer 2016). Die Neubestimmung von Sicherheitsentwürfen, die sich für diese Zeit beobachten lässt, hatte viele Ursachen. Der Fokus dieses Beitrags richtet sich auf zwei Felder des gesellschaftlichen Beziehungsgeflechts, die innerhalb dieses Ursachenbündels zweifellos eine wichtige Rolle spielten: die gesellschaftliche Wohlstandsverteilung und das Geschlechterverhältnis. Beide unterlagen einer Neudefinition und einem Umbruch. Nachdem in den 1950er und frühen 1960er Jahren die Hoffnung verbreitet gewesen war, es werde eine „nivellierte Mittelstandsgesellschaft“ (Schelsky 1953, S. 218) entstehen, in der soziale Unterschiede an Bedeutung verlören, mehrten sich seit den ausgehenden 1960er Jahren und verstärkt nach der Ölpreiskrise von 1973 Stimmen, die eine „neue Armut" oder eine „neue soziale Frage“ (Geißler 1975) diagnostizierten: Soziale Differenzen nahmen in der gesellschaftlichen Wahrnehmung wieder schärfere Konturen an. Die Protestbewegung der späten 1960er Jahre hatte für diese Sensibilisierung erste stärkere Impulse gegeben. Sie stellte überdies das traditionelle Generationenverhältnis in Frage, unmittelbar gefolgt von der zweiten Frauenbewegung, die sich zum Ziel setzte, die herkömmliche Geschlechterordnung aufzubrechen. All diese Entwicklungen provozierten bei vielen Zeitgenoss:innen Unsicherheitsgefühle, stießen eine Neubestimmung von Sicherheitsentwürfen an und veränderten die vorherrschende Sicherheitskultur (zum Begriff der Sicherheitskultur vgl. Daase 2011; Daase 2012). Dies soll anhand von zwei Beispielen nachgezeichnet werden, die in den 1970er Jahren als urbane Sicherheitsprobleme auftauchten, aber auf je unterschiedliche Weise die Wechselbeziehungen sicherheitsbezogener Zugehörigkeitsbestimmungen und Verantwortungshierarchien hervortreten lassen: Die Rede ist vom Großsiedlungsbau und von der Hausbesetzerbewegung. Als Untersuchungsstädte dienen Hamburg und London.

Blickt man auf die Entstehungsgeschichte der Critical Security Studies, die auch in der historischen Sicherheitsforschung eine breite Rezeption erfahren haben, überrascht es, wie selten dieser konstruktivistische Ansatz bislang mit der Frage nach der Konstruktion von Kollektiven verbunden wurde, zumal vor allem eine Zusammenführung mit der konstruktivistischen Nationsforschung naheliegend wäre (vgl. Jutila 2015; Silva 2016 sowie als wenig beachtete frühe Ausnahme Campbell 1992). Diese hätte 
auch Wege aufzeigen können, sich aus der Nationszentriertheit der Critical Security Studies zu lösen, die schon seit ihrer Anfangszeit ein zentraler Kritikpunkt gewesen ist. Denn die Erkenntnis, dass Nationen in Benedict Andersons (2016) Worten „imagined communities“ sind, weist auf ihre Historizität hin und fordert gleichzeitig dazu heraus, sie als eine Form der "gedachten Ordnung" unter anderen zu betrachten. Ungeachtet dessen, dass mit Ole Waever einer der wichtigsten Vertreter der kritischen Sicherheitsforschung durchaus in diese Richtung gedacht hat, verblieben die Anhänger:innen des von ihm maßgeblich entwickelten Theoriemodells der sogenannten Copenhagen School weitgehend bei einem objektivistischen und reifizierenden Identitätsbegriff (vgl. Waever et al. 1993; Waever 1995 sowie die Kritik von McSweeney 1999, S. 74, 404). Waever selbst rekurriert zwar in seinen Ausführungen zu dem Konzept der „societal security“ auf die Nations- und Nationalismusforschung, bezieht sich aber definitorisch in erster Linie auf Anthony D. Smith, das heißt auf einen Theoretiker, der von einem essentialistischen Nationskonzept ausgeht (vgl. Waever et al. 1993; Smith 1999).

Auch die sogenannte PARIS School der kritischen Sicherheitsforschung, die zwar mit ihrem starken Fokus auf Alltagsroutinen vielfach In- und Exklusionsmechanismen - etwa an Grenzen und Flughäfen - untersucht, nimmt sich der Frage nach dem Zusammenhang zwischen Versicherheitlichungsprozessen und Kollektivkonstruktionen kaum an. Vermutlich weil sie in Abgrenzung zur Kopenhagener Schule die Bedeutung von Sicherheitspraktiken betont, stellt auch die PARIS Schule diskursive Aspekte dezidiert in den Hintergrund.

Die sogenannte Aberystwyth oder Welsh School schließlich, das heißt derjenige Zweig der kritischen Sicherheitsstudien, der Gemeinschaft und Identität zu Schlüsselkonzepten der Sicherheitsforschung erklärt, definiert eher normativ, welche Gestalt beide anzunehmen haben, um eine möglichst weitreichende Sicherheit zu gewähren. Dabei gehen ihre Vertreter:innen vom Ideal einer pluralistischen Gesellschaft aus. In bewusster Opposition zur Kopenhagener Schule verstehen sie Sicherheit als positiv $\mathrm{zu}$ bewertende Voraussetzung von Emanzipationsprozessen (vgl. z.B. Booth 2007, S. 111-116). Dieses normative Verständnis und die damit einhergehende Konzentration auf das um Emanzipation bemühte Individuum verstellen bei diesem Ansatz den Blick auf die Ambivalenz, die dem Zusammenhang von Gemeinschaftsvorstellungen und Sicherheitsentwürfen innewohnt. Hier lässt sich eine Leerstelle diagnostizieren, bei der die historische Forschung durch ihre lange Erfahrung mit konstruktivistischen Herangehensweisen wertvolle Impulse liefern kann. 


\section{Soziale Grenzziehungen und Differenzbestimmungen}

Der Großsiedlungsbau, der hier nur für Hamburg betrachtet wird, da er in London für den Wohnungsbau eine weit weniger prägende Rolle besitzt, illustriert vor allem die enge Verzahnung von In- bzw. Exklusionsprozessen mit Sicherheitsdynamiken in exemplarischer Deutlichkeit. Die historische Forschung hat sich mit den Großsiedlungen bereits eingehend auseinandergesetzt, weshalb es ausreicht, sie hier nur knapp auf unsere Fragestellung hin zu analysieren (vgl. Haumann/Heßler 2013; Haumann/Hoschek 2020; Necker 2012). Bis Ende der 1960er Jahre galten sie als Vorzeigeprojekte der modernen Stadtplanung, doch mit der Wende zu den 1970er Jahren wandelte sich ihr Image jäh: Die Modernitätseuphorie und der Machbarkeitsglaube, den sie bis dahin verkörperten, verloren zunehmend an Anhängerschaft, gleichzeitig fanden die Siedlungen auch ästhetisch plötzlich keinen Gefallen mehr. Überdies ließen rasch sichtbar werdende Baumängel die Attraktivität der Siedlungen schwinden. Wer es sich leisten konnte, zog nun zumeist fort. Der Segregationsprozess, der hierdurch eingeläutet wurde, machte in der zeitgenössischen Wahrnehmung die Siedlungen zur Sicherheitsgefahr und ihre Bewohner:innen zur „Problemgruppe“. In Hamburg hatte das beispielsweise zur Folge, dass die Post aus Angst vor Überfällen davon absah, in der Großsiedlung Steilshoop eine Zweigstelle einzurichten. Eine dortige Mietervereinigung beobachtete besorgt, wie die Bewohner:innen der Siedlung über die Sicherheitsbedenken als ein Kollektiv konstruiert wurden. Die Entscheidung der Post, so klagte sie, stigmatisiere „die Bevölkerung von Steilshoop in ihrer Gesamtheit als erhöhtes Sicherheitsrisiko"1.

Wenn Politik- und Medienvertreter:innen die Großsiedlungen immer wieder zur Sicherheitsgefahr stilisierten, konstruierten sie gleichzeitig die Siedlungsbewohner:innen als distinkte Gruppe mit bestimmten sozialen Eigenheiten. Solche Fremdzuschreibungen gehen zwangsläufig mit Selbstbeschreibungen einher; im Falle der Großsiedlungen wurden sie überdies genutzt, um die imaginierte Utopie einer sicheren Gesellschaft zu zeichnen: Die Kritik an der sozialräumlichen Segregation spiegelte hier das Idealbild einer wohlhabenden, gebildeten Gesellschaft, in der soziale „Durchmischung" scharfe Differenzlinien zwischen arm und reich verwischte und die in den Augen der Zeitgenoss:innen auch den sozial Benachteilig-

1 Staatsarchiv Hamburg (StAH) 444-5 Ortsamt Bramfeld, Nr. 209 (Planung, Bau und Unterhaltung der Großsiedlung Steilshoop, Erklärung der Mietergemeinschaft Steilshoop, 19. Januar 1974). 
ten Aufstieg versprach. Die wahrgenommene Unsicherheit ging hier also zunächst von der in den Großsiedlungen sichtbar werdenden sozialen Ungleichheit aus, die in den 1970er Jahren zunehmend problematisiert wurde, und gar nicht von den Bewohner:innen der Großsiedlungen selbst. Doch wurde die Bedrohungswahrnehmung schnell auf sie übertragen.

Dass Kollektivkonstruktionen, die auf einer Fremdzuschreibung beruhen, oftmals mit einer Projektion von Sicherheitsbedrohungen verbunden sind, ist offensichtlich und ließe sich auch für die Hausbesetzerbewegung zeigen - die zweite wahrgenommene Bedrohung der urbanen Sicherheit, die hier zur Untersuchung steht. Für sie soll hier aber vielmehr die Frage im Vordergrund stehen, welche Bedeutung die Aushandlung von Sicherheitsentwürfen bei einem Kollektivbildungsprozess zukam, der nicht oder zumindest nicht primär aus einer Fremdzuschreibung hervorging. Dass der Wert der Sicherheit für die Selbstdefinition der Hausbesetzerbewegungen eine zentrale Rolle spielte, mag auf den ersten Blick überraschen, da diese gewöhnlich mit dem Ziel einer selbstbestimmten Lebensweise assoziiert werden, für die Sicherheitsziele nachrangig erschienen oder mit denen sie sogar als unvereinbar galten. Die Analyse der Hausbesetzerbewegungen in Hamburg und London in den 1970er Jahren wirft Licht auf verschiedene Funktionsweisen von Sicherheitsdiskursen bei der Selbstdefinition von Gruppen, wobei der Vergleich auffällige Unterschiede zwischen beiden Städten zu Tage treten lässt.

Seit den späten 1960er bzw. den frühen 1970er Jahren fanden in vielen europäischen Städten in zunehmender Zahl Hausbesetzungen statt. In London nahm die Hausbesetzerbewegung als eine der ersten ihrer Art 1968 ihren Anfang und entwickelte sich im folgenden Jahrzehnt rasant: Für die zweite Hälfte der 1970er Jahre wird die Zahl der im Englischen als „Squatter" bezeichneten Hausbesetzer:innen hier bereits auf 20.000 bis 35.000 geschätzt (vgl. Kearns 1979, S. 589; Brittain 1975, S. 41). Umfragen zufolge war für $90 \%$ von ihnen der schiere Wohnraummangel ein Motiv, für über $60 \%$ sogar das Hauptmotiv (vgl. Kingham 1977, S. 34-37). Tatsächlich gab es auf dem Londoner Wohnungsmarkt massive Engpässe, so dass in den späten 1970er Jahren um die 200.000 wohnungslose Familien registriert waren (vgl. Kearns 1979, S. 590). Hinzu kamen Paare und Einzelpersonen, deren Zahl nicht erfasst wurde. Der angespannte Wohnungsmarkt erklärt das Spezifikum der Londoner Hausbesetzerbewegung: Ihr gehörten zum einen viele Familien an, zum anderen zahlreiche Migrant:innen, die es aufgrund von Diskriminierung besonders schwer hatten, eine Bleibe zu finden. Seit den frühen 1970er Jahren besetzten dann zunehmend auch Anhänger:innen der alternativen Szene Häuser (Baily 1973, S. 178). Sie verstanden die Hausbesetzungen vielfach als politischen 
Protest, gleichzeitig ging es ihnen oftmals auch um das damit verbundene Gemeinschaftserlebnis. Unter den Londoner Hausbesetzer:innen blieb diese Gruppe allerdings die Minderheit.

In Hamburg sah die Situation anders aus: Die Einwohnerzahl der Hansestadt war seit Ende der 1960er Jahren rückläufig. Obwohl die Ansprüche in Bezug auf die Wohnfläche pro Person stiegen und vor allem viele der sogenannten „Gastarbeiter" noch in überfüllten Sammelunterkünften lebten, lässt sich von einem wirklichen Wohnraummangel für Hamburg in den 1970er Jahren nicht sprechen. Dies spiegelte sich in den Zielen der Hausbesetzer:innen wider. Ihre Absicht war es, einerseits gegen Immobilienspekulation sowie gegen die städtische Wohnungsbaupolitik und die Gentrifizierung der Innenstadt zu protestieren und sich anderseits Raum für einen selbstbestimmten Lebensstil zu schaffen. In Hamburg wurde die Bewegung ausschließlich von Anhänger:innen des linksalternativen Milieus getragen. Die Gruppe war mit mehreren Hundert Hausbesetzer:innen deutlich kleiner als in London. Ihr gehörten vor allem junge Männer an, Familien hingegen zählten erst in den 1980er Jahren in sehr geringer Anzahl dazu. ${ }^{2}$

Die Unterschiede zwischen den Hausbesetzerbewegungen in Hamburg und London spiegeln sich in ihrer Selbstpositionierung als Kollektiv und den damit einhergehenden Sicherheitsentwürfen. In London verorteten sich die „Squatter“ gern in einer jahrzehnte-, wenn nicht gar jahrhundertealten britischen Tradition. In Squatting. The Real Story, der wohl ausführlichsten Selbstdarstellung der britischen Hausbesetzer:innen, wurden nationale Kontinuitätslinien gezogen, die bis ins Mittelalter zurückreichen (vgl. Ward 1980, S. 104-109; Ward 2002). Einen besonders wichtigen Stellenwert nahm für die historische Selbstverortung die Hausbesetzerbewegung der unmittelbaren Nachkriegszeit ein (vgl. Friend 1980). An ihr waren vor allem Veteranen beteiligt gewesen, die nicht zuletzt als Rekompensation für ihren Kriegseinsatz das Recht auf eine Wohnung einklagten. Der Verweis auf die Kriegshelden versprach für die „Squatter“ der 1970er Jahre offenbar Prestige. Überdies definierten sie sich damit eindeutig als Teil einer nationalen Solidargemeinschaft.

Dieses Zugehörigkeitsbekenntnis der britischen Hausbesetzer:innen mag auch durch die Tatsache motiviert gewesen sein, dass sie in Teilen der britischen Gesellschaft als Bedrohung der öffentlichen Ordnung betrachtet wurden. Um einer solchen Wahrnehmung den Wind aus den Segeln neh-

2 Vgl. https://youtu.be/146v6DRIelM, letzter Zugriff: 25.3.2021; Borgstede 2019; Amatine 2011, S. 112-113. 
men, bemühten sich die „Squatter“ gezielt darum, ein anderes Bild von sich selbst zu etablieren. Mit einem Plakat aus dem Jahr 1975 beispielsweise bemühten sie sich, davon zu überzeugen, dass sie mit der Instandsetzung vom Verfall bedrohter Häuser die Brandgefahr senken würden (vgl. Abb. 1). Nicht als Bedrohung, sondern als Garanten der Sicherheit wollten sie also gelten.

\section{the case for squatters}

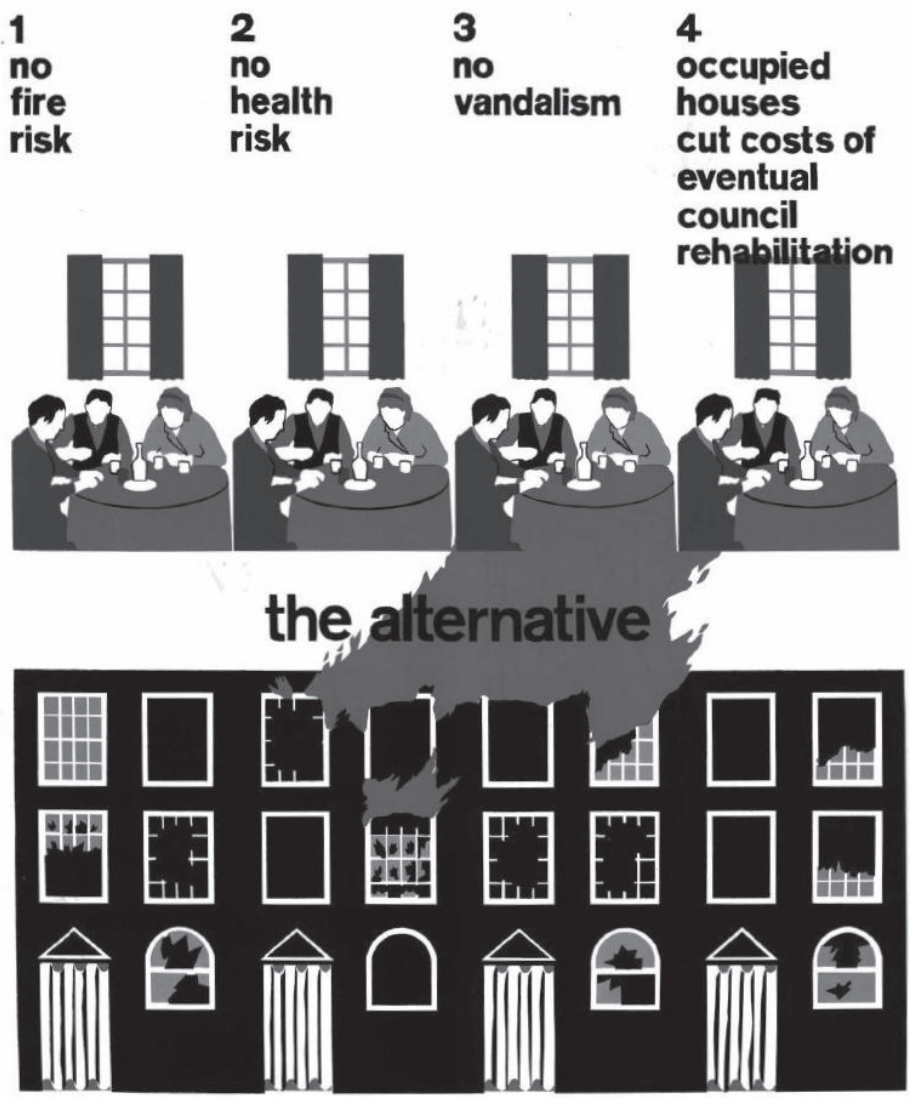

protest against council's harrassment of squatters picket islington town hall tues. 29 july 6.00.p.m.

Abb. 1: Pro-squatting Poster aus Islington, London, 1975 [Courtesy of Interference Archive] (aus: Finchett-Maddock 2014, S. 210) 
Die Hamburger Hausbesetzer:innen positionierten sich auf ganz andere Weise innerhalb des größeren gesellschaftlichen Kollektivs. Auch sie interpretierten ihre Aktivitäten im Lichte der nationalen Geschichte: Dazu stellten sie sich gewöhnlich in die Tradition des Widerstandes gegen den Nationalsozialismus (vgl. Krüger 2021). Immer wieder bezichtigten sie den Hamburger Senat, die Bundesrepublik oder die Medien nationalsozialistischer Methoden. Ein Flugblatt verstieg sich sogar dazu zu behaupten, die bürgerliche Presse überbiete „die Hetze, wie sie die Faschisten im 3.

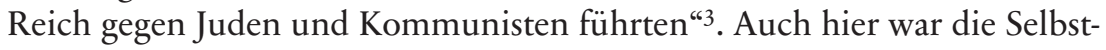
deutung also fest im nationalen Rahmen verankert, machte allerdings politische Differenzkategorien stark, anstatt an die nationale Gemeinschaft und Solidarität zu appellieren, wie dies die Londoner „Squatter" taten.

\section{Geschlechtsspezifische Verantwortungshierarchien}

Wenn die vorwiegend männlichen Hamburger Hausbesetzer die Notwendigkeit der Selbstverteidigung beschworen, während die Londoner Bewegung zu einem hohen Anteil von Familien getragen wurde, die ihr Anrecht auf Solidarität und ihre Schutzbedürftigkeit hervorhoben, so knüpften beide an die traditionelle Verantwortungsverteilung auf dem Feld der Sicherheit an. Deren Wirkmacht ist nur durch ihre lange historische Persistenz zu erklären, und für ihr Verständnis lohnt ein kurzer Rückblick ins 19. Jahrhundert.

Die geschlechtsspezifische Bestimmung von Sicherheitsverantwortung, so die hier vertretene These, war über lange Zeit konstitutiv für die Konstruktion von Sicherheitskollektiven. Auch dieser Zusammenhang ist in der kritischen Sicherheitsforschung bislang kaum beachtet worden. Diese hat sich Genderfragen bislang nur selten angenommen und sich überdies oftmals darauf beschränkt, Frauen als Referenzobjekte in Versicherheitlichungsprozessen in den Blick zu nehmen (z.B. Hansen 2000). Eine solche Analyseperspektive liegt nahe, ist aber letztlich ebenfalls eine Konsequenz historisch langlebiger geschlechtsspezifischer Verantwortungshierarchien.

Sicherheit galt traditionell als genuin maskuliner Handlungsbereich. Im 19. Jahrhundert verfestigte sich diese Vorstellung mit der Herausbildung der bürgerlichen Werteordnung einerseits und dem Aufkommen der Nationalstaaten und dem mit ihnen einhergehenden Gedanken der (männlich geprägten) Wehrgemeinschaft andererseits. Kaum jemand stellte in

3 Archiv des Hamburger Instituts für Sozialwissenschaft, SBe 600. 
Frage, dass Sicherheitsbelange in den Verantwortungsbereich der Männer fielen. Begründet wurde diese Zuschreibung gewöhnlich mit zwei Argumenten, die von vermeintlich biologischen und damit absolut gesetzten Geschlechterunterschieden ausgingen. Zum einen wurde physische Stärke ins Feld geführt: Frauen als „schwachem Geschlecht“ wurde die Fähigkeit, für Sicherheit zu sorgen, aufgrund ihrer geringeren Körperkraft abgesprochen. Zum anderen war die Vorstellung verbreitet, dass Frauen nicht über die nötigen kognitiven Fähigkeiten verfügten, die für das Verständnis von und den Umgang mit Sicherheitsfragen notwendig seien. Insbesondere fehle ihnen die Kompetenz, über den Moment und das Individuum hinauszudenken, was für sicherheitsrelevante Zukunftsprognosen ebenso wichtig erschien wie für das Verständnis komplexer gesellschaftliche $\mathrm{Zu}$ sammenhänge (vgl. Hausen 1976).

Diese Vorstellung legitimierte das Konzept der „zwei Sphären“, das sich wirkmächtig im 19. Jahrhundert durchsetzte (vgl. Greven-Aschoff 1981, S. 22-43). Frauen sollten sich diesem Modell zufolge vor allem in der Sphäre des Privaten und der Familie bewegen, in der ihr Aufgabenfeld liege. Die Sphäre der Öffentlichkeit hingegen - und hier dachte man vor allem an Politik und Erwerbsarbeit - sollte den Männern vorbehalten bleiben. Eng damit verknüptt war eine ausgeprägte Mütterlichkeitsideologie, die ebenfalls die sicherheitsbezogene Verantwortungsverteilung determinierte: Als Mütter galten Frauen als schutzbedürftig, den Männern kam damit die Rolle der Beschützer zu. Tatsächlich betrachteten viele Zeitgenoss:innen just den Moment, in dem Frauen und Kinder in Gefahr gerieten, als den Moment, der eine existenzielle Bedrohungssituation markierte und Sicherheitsmaßnahmen erforderlich machte.

Die den Männern zugeschriebene Verpflichtung, Schutz zu garantieren, umfasste die als männliche Tugend geltende Bereitschaft, die eigene individuelle Sicherheit für die Sicherheit des Kollektivs aufzugeben. Die Aufforderung, „Mannhaftigkeit“" zu beweisen, konnte daher etwa dazu dienen, die Soldaten zum Kampf zu motivieren. Gerade im Falle einer Bedrohung der physischen Existenz, wie sie der Krieg darstellte, sollte der Appell an eine als männliche Pflicht angesehene Beschützerrolle den für notwendig erachteten Gruppenzusammenhalt stärken. Die wahrgenommene Schutzbedürftigkeit bestimmter Gruppen innerhalb einer größeren Gemeinschaft war eine starke Antriebskraft für diejenigen, die im Krieg zu kämpfen hatten. Nicht nur das Streben nach Sicherheit allgemein konnte also ein zentrales Movens für die Konstruktion kollektiver Identität sein. Vielmehr trug gleichermaßen auch die Verteilung von Sicherheitsverantwortung entscheidend dazu bei: Sicherheitshierarchien waren für die Imagination des Sicherheitskollektivs konstitutiv. 
Zwar mag es so erscheinen, dass der Wert der „Mannhaftigkeit“ oder der „männlichen Ehre“ in manchen Situationen - als Beispiel wäre hier das Duell zu nennen - demjenigen der „Sicherheit“ übergeordnet wurde. Eine solche Interpretation vereinfacht aber zum einen in der scharfen Gegenüberstellung dieser Werte deren komplexes Wechselverhältnis, zum anderen vernachlässigt es die Wandelbarkeit der Definition von Sicherheit. Denn wenn „Ehre“ und „Manneswürde“ auf dem Spiel standen, wurde dies von den Zeitgenossen durchaus als existenzielle Gefahr für die kollektive Sicherheit wahrgenommen. Da große Gemeinschaften nur als ,imagined communities" bestehen können, werden gemeinsame Werte für den inneren Zusammenhalt in der Regel als unverzichtbare Existenzbedingung angesehen. Solche Gemeinschaften nehmen nicht nur diejenigen Situationen als existenzielle Bedrohung wahr, in denen die physische Existenz ihrer Mitglieder auf dem Spiel steht. Gefährlich scheinen ihnen auch Situationen, in denen die Kohäsionskräfte zu schwinden scheinen, denen der innere Zusammenhalt zugeschrieben wird. Ganz ähnlich argumentiert Ole Weaver mit seinem Konzept der „societal security“ (vgl. Waever et al. 1993; Waever 1995). Unter diesen Kohäsionskräften nahmen im 19. Jahrhundert die Werte der Mannhaftigkeit und Ehre einen wichtigen Platz ein.

Gleichzeitig bestimmten die sicherheitsbezogenen Verantwortungshierarchien in starkem Maße gesellschaftliche Machtverhältnisse. Machtansprüche wurden mit dem Hinweis auf die Bürde gerechtfertigt, die mit der Sicherheitsverantwortung zu tragen sei, Unterordnung mit dem Hinweis auf die vermeintliche Unfähigkeit, Sicherheitsfragen angemessen zu beurteilen und zu behandeln. Auf diese Weise wurden mit der Verteilung der Sicherheitsverantwortung Differenzlinien geschaffen, die die ursprüngliche Vorstellung einer Zusammengehörigkeit leicht in einen antagonistischen Gegensatz verwandeln konnten. Wie schnell die sicherheitsbezogenen Differenzkategorienpaare gefährlich/ungefährlich und schutzbedürftig/für den Schutz verantwortlich ineinanderfließen konnten, soll nun noch einmal am Beispiel der Großsiedlungen und der Hausbesetzerbewegungen in Hamburg und London während der 1970er und frühen 1980er Jahre genauer in den Blick genommen werden.

\section{Gefäbrdet oder gefährlich?}

Für Frankfurt und Berlin hat Sven Reichardt in seiner Studie zum linksalternativen Milieu die spezifischen Männlichkeitsvorstellungen der Hausbesetzer herausgearbeitet und diese als Motiv für deren hohe Gewaltbereit- 
schaft interpretiert (vgl. Reichardt 2010, S. 498-571). Obzwar die für diesen Beitrag gesichteten Quellen für die Hamburger Hausbesetzungen der 1970er und frühen 1980er Jahre eine solche Deutung nicht explizit belegen, ist die in dem Milieu zur Schau gestellte Militanz (Abb. 2) doch auch im Kontext der traditionellen Verantwortungshierarchien zu analysieren, die in starkem Maße aus dem spätestens seit dem 19. Jahrhundert vorherrschenden Geschlechterrollenmodell hergeleitet wurden. In den besetzten Häusern der Hafenstraße wurde dies in den 1980er Jahren bereits kritisch diskutiert. Der Einrichtung eines eigenen Hauses nur für Hausbesetzerinnen 1987 lag als ein wichtiges Motiv auch zugrunde, dass die Frauen die hohe Gewaltbereitschaft ablehnten, die sie als männliches Rollenmuster deuten (vgl. Amatine 2011, S. 106-112). Die Kritik der Frauen führte dazu, dass einige männliche Hausbesetzer ihre Verhaltensweisen kritisch reflektierten. $\mathrm{Ob}$ die Infragestellung der traditionellen Geschlechterrollen aber insgesamt ein allmähliches Abrücken von der Militanz beförderte oder vielmehr Verunsicherung auslöste, die sie vielleicht noch verstärkte, bleibt eine offene Forschungsfrage, die sich auf der Grundlage der für diesen Beitrag gesichteten Quellen nicht beantworten lässt.

Aus der Vergleichsperspektive heraus ist wichtig, dass die hohe Gewaltbereitschaft der Hamburger Hausbesetzerbewegung diese deutlich von ihrem Londoner Gegenstück unterschied. Schon neun Jahre vor dem Konflikt um die 1982 besetzten Häuser in der Hafenstraße kam es in der Hansestadt zu gewaltsamen Zusammenstößen mit der Polizei (vgl. Führer 2016, S. 175-187). Diese Radikalisierung ist sicherlich maßgeblich auf die in der Bundesrepublik generell höhere Gewaltbereitschaft von Teilen der linksgerichteten Protestbewegung zurückzuführen. Vor allem die hohe ideologische Aufladung liegt hier als Ursache nahe. Da sich die Hamburger Hausbesetzer:innen wie oben dargelegt zur Selbstverteidigung berechtigt, ja gezwungen, sahen, nahmen sie es in Kauf oder legten es gar darauf an, selbst als Sicherheitsgefahr wahrgenommen zu werden. Dass traditionelle Männlichkeitsvorstellungen sie hier befeuert haben mögen, wie Reichardt das für Berlin und Frankfurt argumentiert, liegt auch für die überwiegend aus jungen Männern bestehende Gruppierung in Hamburg nahe.

Verstärkt wurde der Unterschied zwischen der Londoner und der Hamburger Bewegung überdies wohl durch eine divergierende Rechtslage: In Großbritannien waren Hausbesetzungen eine zivilrechtliche Angelegenheit, aber kein Straftatbestand (vgl. Ad Hoc Group to Produce the Fifth Edition of the Squatters' Handbook 1978, S. 4-8). Dies erschwerte und verzögerte die Räumung, sofern diese von den Hausbesitzer:innen angestrebt wurde. In der Bundesrepublik hingegen galten Hausbesetzungen als Haus- 


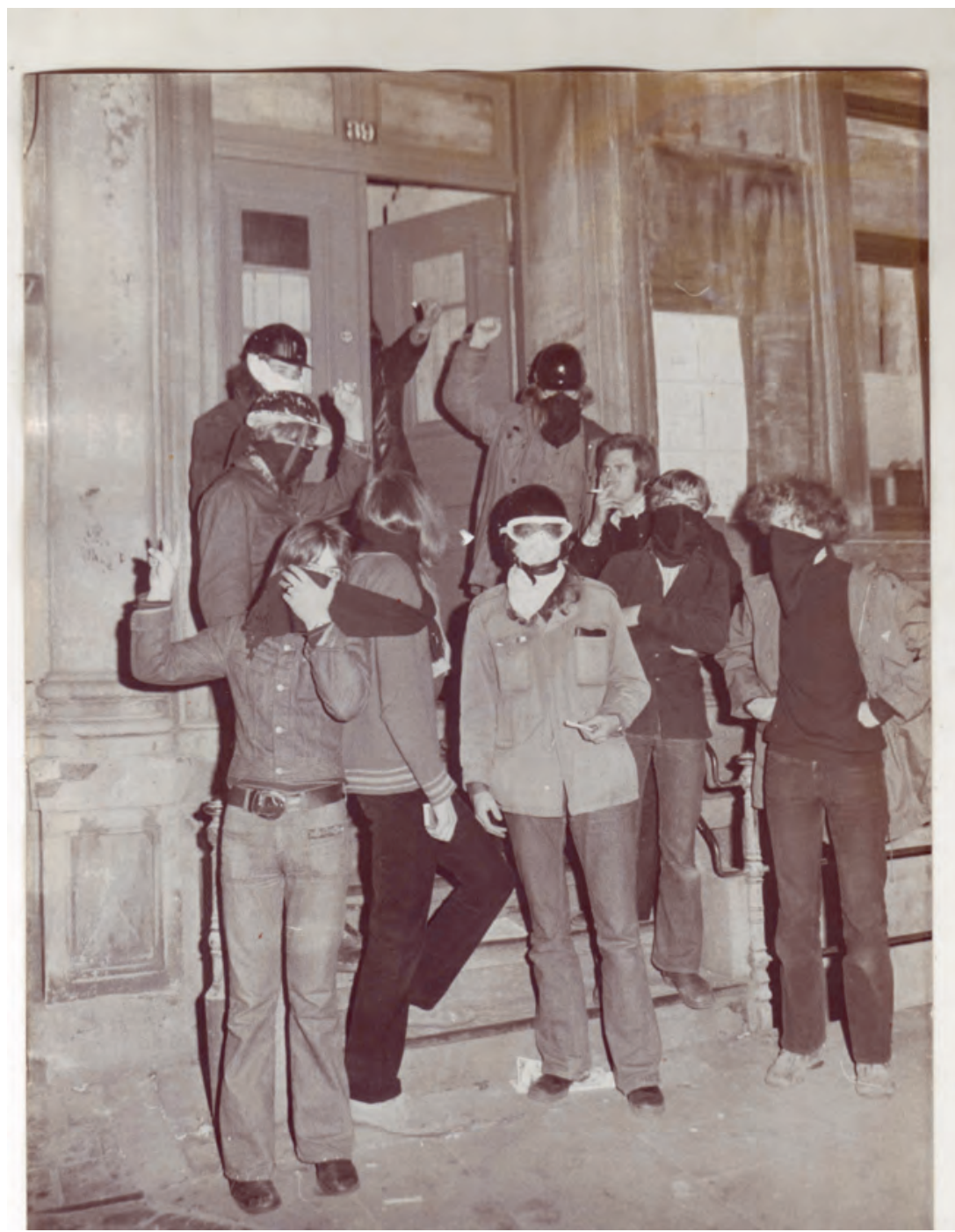

Abb. 2: Hausbesetzung Ekhofstraße, Gruppenfoto, Mai 1975 (mit freundlicher Genehmigung der Hamburger Morgenpost)

friedensbruch und damit als Straftat. Zwar kam es auch hier deshalb nicht immer zwangsläufig zu einer Räumung (vgl. Führer 2016, S. 154-155). Doch mussten die Besetzer:innen stets mit einem möglichen Einschreiten der Polizei rechnen. In ihrer grundsätzlich oppositionellen Haltung waren 
die Hamburger Hausbesetzer:innen bereit, dieses Risiko auf sich zu nehmen. Demgegenüber lässt sich vermuten, dass die fehlenden Familien unter den Hamburger Hausbesetzer:innen unter anderem auf eine geringere Risikobereitschaft vor allem von Eltern kleiner Kinder zurückzuführen ist.

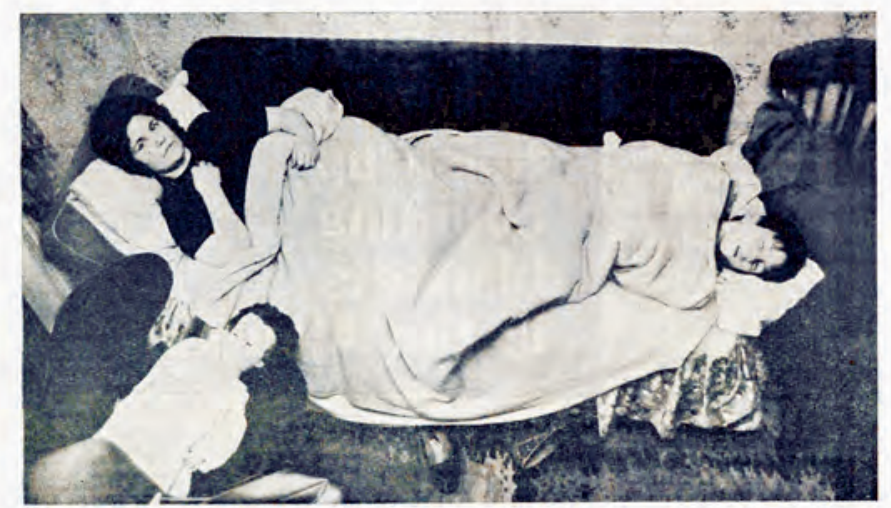

Bedtime for squatters: Moggie O'Shannon and son John.

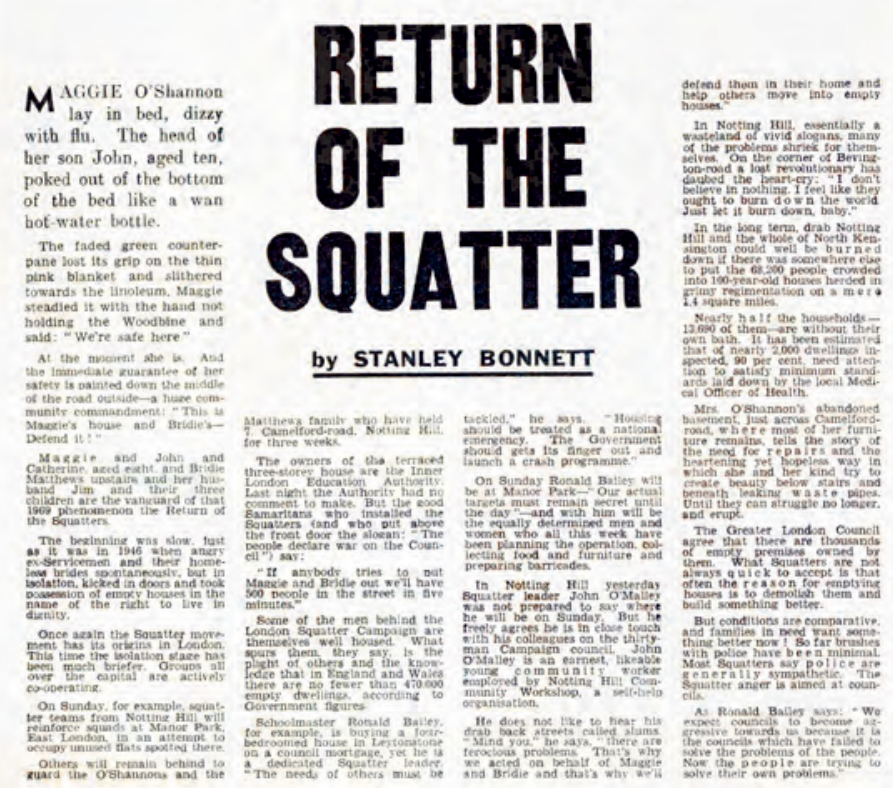

Abb. 3: Stanley Bonnett, „Return of the Squatter“, in: Daily Mirror, 7.2.1969, S. 13 (C) Daily Mirror) 


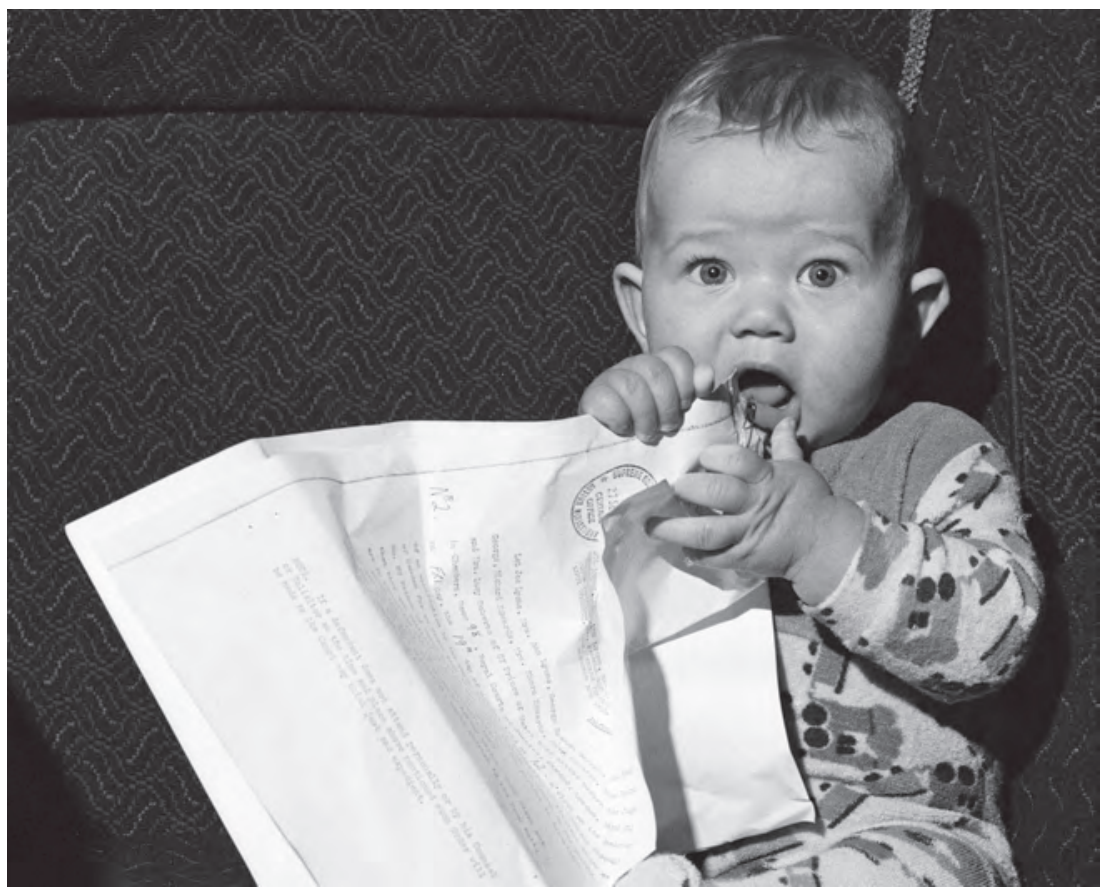

Abb. 4: "Question: What did baby Mark do when he was served with a writ? Answer: He ate it!", so die Bildüber-und-unterschrift zu diesem Foto, das der Daily Mirror am 29. Dezember 1973 großformatig druckte. (C) Daily Mirror)

Die Londoner „Squatter“ verfolgten einen gänzlich anderen Sicherheitsentwurf, und auch dies stand im Zusammenhang mit traditionellen Geschlechter- und Familiennormen. Sie waren bemüht, das öffentliche Bild ihrer Bewegung durch Fotos von Frauen und Kindern zu prägen und auf diese Weise ihre Schutzbedürftigkeit hervorzuheben. Der linksgerichtete Daily Mirror etwa brachte Anfang 1969 das Bild einer grippekranken Mutter mit ihrem zehnjährigen Sohn und zitierte ihre Erleichterung nach der Hausbesetzung: „We are safe here.“ (Abb. 3) Im September 1973 zeigte dasselbe Blatt ein großformatiges Foto eines sechs Monate alten Säuglings einer "Squatter"-Familie, der an der Auszugsanweisung nuckelte (Abb. 4). Auch in Selbstdarstellungen zeigten die Hausbesetzer:innen immer wieder Bilder von Familien mit kleinen Kindern. Ähnlich wie mit der Konstruktion einer Traditionslinie zu den Hausbesetzer:innen der unmittelbaren Nachkriegszeit unterstrichen sie auch mit dem Verweis auf die Schutzwür- 
digkeit ihrer Familien ihre Zugehörigkeit zur nationalstaatlichen Solidargemeinschaft. Gleichzeitig betonten sie damit, dass sie die traditionellen Familiennormen befolgten und hochschätzten. Zur gesellschaftlichen Verunsicherung, die die Infragestellung der herkömmlichen Geschlechterordnung insbesondere seitens der zweiten Frauenbewegung hervorrief, wollten sie nicht beitragen. Vor allem aber grenzten sie sich damit von den jugendlichen Hausbesetzer:innen ab, die vor allem nach dem nötigen Freiraum suchten, um aus den Zwängen bürgerlicher Normen auszubrechen. Als die Londoner „Squatter“-Szene sich im Laufe der 1970er Jahre diversifizierte und eine immer größere Anzahl von Hausbesetzer:innen, inspiriert durch die jugendliche Protestbewegung, vor allem dem Motiv folgte, eine alternative Lebensweise auszuprobieren und mit neue Formen des Gemeinschaftslebens zu experimentieren, erregte dies bei den „Family Squatters" starke Abwehrreaktionen. Einige Beobachter sprachen gar von einem „Schisma“ der Bewegung (Plate 1984, S.31). Viele „Family Squatters“ fürchteten, dass der gute Ruf der Bewegung in Gefahr gerate. Diese Sorge war nicht unberechtigt, denn in den Medien wurden die "Squatter" nun zunehmend mit Sexparties, Alkoholexzessen, Drogenkonsum und einer latenten Gewaltbereitschaft in Verbindung gebracht (vgl. Kearns 1979, S. 591). Die „Family Squatter“ wollten als harmloser und schutzbedürftiger Teil der Gesellschaft betrachtet werden, nicht als Dropouts, die sich von dieser abzugrenzen suchten und daher von ihr leicht als Bedrohung wahrgenommen werden konnten.

Die zähen Bemühungen der „Family Squatter“, sich als schutzbedürftiger Teil der Gesellschaft zu präsentieren, deuten bereits die Schwierigkeiten an, auf die sie dabei stießen. Diese lagen darin begründet, dass in der Fremdwahrnehmung oft nur ein kleiner Schritt notwendig ist, mit dem die Zuschreibung der Gefährdetheit in diejenige der Gefährlichkeit umschlägt. Und um dies zu illustrieren, lohnt sich ein nochmaliger Blick auf das Beispiel der Großsiedlungen in der Bundesrepublik beziehungsweise hier speziell in Hamburg. Der Großsiedlungsbau war seit seinen Anfängen in starkem Maße mit dem sozialen Wohnungsbau und somit auch mit dem Gedanken einer gesellschaftlichen Solidaritätsverpflichtung verbunden gewesen. Die Vorstellung, dass hier besonders vulnerable und schutzbedürftige gesellschaftliche Gruppen leben würden, verfestigte sich in der medialen Öffentlichkeit in den 1970er Jahren aber nicht zuletzt auch dadurch, dass die als reine Wohnkomplexe fern der Arbeitsstätten geplanten Siedlungen tagsüber vor allem von Frauen und Kindern belebt wurden. Daher lenkte die Berichterstattung, zumal wenn sie fotografisch unterlegt war, ihren Fokus fast exklusiv auf sie. Als Beispiel mag hier ein Artikel von Gisela Stelly und Stefan Aust in der Wochenzeitung Die Zeit 
dienen, der 1971 die Wohnerfahrungen der Frauen in der Hamburger Großsiedlung Steilshoop thematisierte. Wenn sie von einer „Wohnstadt, die so festungsgleich wirkt und doch nicht schützt" schrieben, implizierten sie die Schutzwürdigkeit der Bewohner:innen (Aust/Stelly 1971). Mit der Einschätzung, dass das Schutzversprechen nicht eingelöst werden könne, trafen Aust und Stelly den Tenor der Zeit. Damit wurden aus zeitgenössischer Sicht zunächst die Siedlungen zu bedrohlichen Orten, deren Bedrohlichkeit schließlich auch auf diejenigen projiziert wurde, deren Schutz ursprünglich gefordert worden war.

In besonderem Maße galt dies für die Kinder, die in den Großsiedlungen aufwuchsen. Immer wieder wurde ihre Situation in der medialen Öffentlichkeit problematisiert. Wenn ihre Gefährdung beschrieben wurde, barg dies immer schon die Angst vor einer zukünttigen Bedrohung: Tatsächlich vollzogen sie der verbreiteten Wahrnehmung zufolge mit dem Erreichen des Jugendalters auch den Übertritt aus einer als schützenswert angesehenen in eine potenziell als gefährlich eingeschätzte Gruppe. Dass Kriminalitätsstatistiken keinerlei Hinweise auf eine erhöhte Jugendkriminalität in den Siedlungen lieferten, konnte diese Vorstellung der Zeitgenoss:innen nicht korrigieren (vgl. Weinhauer 2013).

Dass gerade die Wahrnehmung von Heranwachsenden sehr schnell von bedroht in bedrohlich umschlagen konnte, ist gewiss kein Zufall. Speziell für die 1970er Jahre mag eine besorgte Aufmerksamkeit gegenüber der Jugend durch die Jugendproteste und das allgemein ins Wanken geratene Generationenverhältnis besonders ausgeprägt gewesen sein. Aber die Sorge um die Jugend stand in einer längeren Tradition und entwickelte gerade in Zeiten großer gesellschaftlicher Unsicherheit eine besondere Wirkmacht. Denn wo Sicherheit verhandelt wird, wird immer auch die Zukunft behandelt (vgl. Henne et al. 2018): Die Bedrohung, die durch Sicherheitsmaßnahmen abgewehrt werden soll, ist ein für die Zukunft prophezeites mögliches Übel. Die Jugend aber gilt als „Verkörperung der Zukunft“ und wird damit zur Projektionsfläche für Sicherheitsprognosen (Speitkamp 1998, S. 130).

\section{Fazit}

Indem Sicherheitsappelle Zugehörigkeit definieren, stecken sie zwangsläufig gesellschaftliche Grenzen ab. Sie fungieren auf diese Weise in hohem Maße als Katalysator für die Konstruktion eines kollektiven Selbstbildes und damit auch von Differenzkategorien, wie das Beispiel der Großsiedlungsbewohner:innen Hamburgs sehr eindrücklich belegt. Deutlich wurde 
auch, dass nicht nur die Verfolgung von Sicherheitszielen die Gruppenbildung bedingt, sondern zwischen beiden eine enge Wechselbeziehung herrscht. Allgemein verändern Gruppenbildungsprozesse oft verbreitete (Un-)Sicherheitswahrnehmungen. So führen die Bemühungen um Gruppenbildung oder um die Stärkung des Zusammenhalts einer Gruppe leicht dazu, dass Bedrohungsszenarien ausgemalt und Versicherheitlichungsprozesse in Gang gesetzt werden. Hieraus erklärt sich wohl zumindest zu einem Teil die ausgeprägte Sicherheitssemantik der Hamburger Hausbesetzer:innen.

Die Londoner Hausbesetzer:innen, die nicht darauf zielten, sich nach außen hin abzugrenzen, sondern die vielmehr ihre Integration in die nationale Solidargemeinschaft anstrebten, zogen hingegen ein anderes Register sicherheitsbezogener Differenzkategorien, indem sie die Schutzwürdigkeit ihrer Familien betonten. Diese Strategie ging jedoch nur teilweise auf, denn es blieb eine Frage der Definition, ob die Differenzkategorien, die sie aufriefen, aus unterschiedlichen Positionen in der sicherheitsbezogenen Verantwortungshierarchie entstanden oder ob sie einen Antagonismus bargen: Aus dem Mit- oder Nebeneinander konnte schnell ein Gegeneinander werden. Dies zeigt sich deutlich am Beispiel der Wahrnehmung von Kindern und Jugendlichen in den Großsiedlungen. Um Dynamiken der Sicherheit zu verstehen, so lässt sich bilanzieren, kann die Analyse der Wechselbeziehungen zwischen den verschiedenen Arten sicherheitsbezogener Differenzkategorien und der mit ihnen zusammenhängenden Kollektivkonstruktionen einen entscheidenden Beitrag leisten.

\section{Literatur}

Ad Hoc Group to Produce the Fifth Edition of the Squatters' Handbook (1978): Squatters' Handbook.

Amatine (2011): Gender und Häuserkampf. Münster: Unrast.

Anderson, Benedict (2016): Imagined Communities. Reflections on the Origin and Spread of Nationalism. London: Verso.

Bailey, Ron (1973): The Squatters. Harmondsworth: Penguin Books.

Booth, Ken (2007): Theory of World Security. Cambridge: Cambridge University Press.

Borgstede, Simone Beate (2010): Der Kampf um die Herzen die Köpfe der Menschen. In: Das Argument 52, H. 289, S. 849-858.

Brittain, Victoria (1975): Squatters' Rights and Wrongs. In: The Illustrated London News, 01.08.1975, S. 41-43. 
Conze, Eckart (2017): Geschichte der Sicherheit. Entwicklung - Themen - Perspektiven. Göttingen: Vandenhoeck \& Ruprecht.

Daase, Christopher (2011): Sicherheitskultur - Ein Konzept zur interdisziplinären Erforschung politischen und sozialen Wandels. In: Sicherheit und Frieden 29, S. 59-139.

Daase, Christopher et al. (Hrsg.) (2012): Sicherheitskultur. Soziale und politische Praktiken der Gefahrenabwehr. Frankfurt am Main, New York: Campus.

Finchett-Maddock, Lucy (2014): Squatting in London. Squatters' Rights and Legal Movement(s). In: Steen, Bart (Hrsg.): The City is Ours. Squatting and Autonomous Movements in Europe from the 1970s to the Present. Oakland: PM Press, S. 207-231.

Friend, Andrew (1980): The Post War Squatters. What happened when there were no Homes for the World War Two Heroes. In: Anning, Nick: Squatting. The Real Story. London: Blackrose Press, S. 110-119.

Führer, Karl Christian (2016): Die Stadt, das Geld und der Markt. Immobilienspekulation in der Bundesrepublik 1960-1985. Berlin: De Gruyter.

Geißler, Heiner (1980): Die Neue Soziale Frage. Analysen und Dokumente. Freiburg im Breisgau: Herder Verlag.

Geyer, Martin (2016): Die neue Wirklichkeit von Sicherheit und Risiken: Wie wir mit dystopischen, utopischen und technokratischen Diagnosen von Sicherheit zu leben gelernt haben. In: Leendertz, Ariane/Meteling, Wencke (Hrsg.): Die neue Wirklichkeit: semantische Neuvermessungen und Politik seit den 1970erJahren. Frankfurt am Main, New York: Campus, S. 281-315.

Greven-Aschoff, Barbara (1981): Die bürgerliche Frauenbewegung in Deutschland 1894-1933. Göttingen: Vandenhoeck \& Ruprecht.

Hansen, Lene (2000): The little Mermaid's silent Security Dilemma and the Absence of Gender in the Copenhagen School. In: Millennium. Journal of International Studies 29, H. 2, S. 285-306.

Haumann, Sebastian/Heßler, Martina (Hrsg.) (2013): Westeuropäische Großsiedlungen. Berlin: Deutsches Institut für Urbanistik (Informationen zur modernen Stadtgeschichte).

Haumann, Sebastian/Hoschek, Swenja (Hrsg.) (2020): Großsiedlungen als Problemkonstruktion. Stuttgart: Forum Stadt Verlag.

Hausen, Karin (1976): Die Polarisierung der „Geschlechtscharaktere“. Eine Spiegelung der Dissoziation von Erwerbs- und Familienleben. In: Conze, Werner (Hrsg.): Sozialgeschichte der Familie in der Neuzeit Europas. Neue Forschungen. Stuttgart: Klett, S. 363-393.

Henne, Sebastian/Kampmann, Christoph/Marciniak, Angela/Meteling, Wencke (2018): „Security turns its eye exclusively to the future“. Zum Verhältnis von Sicherheit und Zukunft in der Geschichte. In: Kampmann, Christoph/Marciniak, Angela/Meteling, Wencke (Hrsg.), „Security turns its eye exclusively to the future". Zum Verhältnis von Sicherheit und Zukunft in der Geschichte. Baden-Baden: Nomos Verlag, S. 9-36. 
Jeismann, Michael (1992): Das Vaterland der Feinde. Studien zum nationalen Feindbegriff und Selbstverständnis in Deutschland und Frankreich 1792-1918. Stuttgart: Klett-Cotta.

Jutila, Matti (2015): Securitization, History, and Identity: some conceptual Clarifications and Examples from Politics of Finnish War History. In: Nationalities Papers 436, S. 927-943.

Kearns, Kevin C. (1979): Intraurban Squatting in London. In: Annals of the Association of American Geographers 69, H. 4, S. 589-598.

Kingham, Mike (1977): Squatters in London. Institute of Community Studies/Shelter. National Campaign for the Homeless. London: Shelter.

Krüger, Christine G. (2020): Von Mümmelmannsberg bis Allermöhe: Sicherheitsund Unsicherheitskonstruktionen. In: Forum Stadt 47, H. 3, S. 223-237.

Krüger, Christine G. (2022): Risky Housing: Squatting in the 1970s and early 1980s. In: Geyer, Martin (Hrsg.): Places of Risk - Sites of Modernity: Cultures of Security and Risk since the 1970s. New York: Berghahn (i.E.).

McSweeney, Bill (1999): Security, Identity and Interests. A Sociology of International Relations. Cambridge: Cambridge University Press.

Necker, Sylvia (2012): Hässlich, aber innovativ? Architektur und soziale Wirklichkeit der Großsiedlung Steilshoop. In: Forschungsstelle für Zeitgeschichte Hamburg (Hrsg.): 19 Tage Hamburg. Ereignisse und Entwicklungen der Stadtgeschichte seit den fünfziger Jahren. München: Dölling und Galitz, S. 160-173.

Plate, Steve (1980): A Decade of Squatting. The Story of Squatting in Britain since 1968. In: Anning, Nick: Squatting. The Real Story. London: Blackrose Press, S. 14-103.

Reichardt, Sven (2014): Authentizität und Gemeinschaft. Linksalternatives Leben in den siebziger und frühen achtziger Jahren. Berlin: Suhrkamp.

Silva, Marta (2016): Securitization as a Nation-Building Instrument. In: IAPSS Politikon 29, S. 201-214.

Schelsky, Helmut (1953): Wandlungen der deutschen Familie in der Gegenwart. Darstellung und Deutung einer empirisch-soziologischen Tatbestandsaufnahme. Dortmund: Ardey Verlag.

Smith, Anthony D. (1999): National Identity. London: Penguin Books.

Speitkamp, Winfried (1998): Jugend in der Neuzeit. Göttingen: Vandenhoeck \& Ruprecht.

Stelly, Gisela/Aust, Stefan (1971): Mein Heim, meine Zelle. Protokolle aus einer Großstadt-Mustersiedlung. In: Die Zeit, Nr. 8 (19.2.1971).

Ward, Colin (1980): The early Squatters. Squatting from the Middle Ages to the Second World War. In: Anning, Nick: Squatting. The Real Story. London: Blackrose Press, S. 104-109.

Ward, Colin (2002): Cotters and Squatters. Housing's hidden History. Nottingham: Five Leaves.

Waever, Ole/Buzan, Barry/Carlton, David (1993): Identity, Migration and the New Security Agenda in Europe. London: Pinter. 
Waever, Ole (1995): Identity, integration and security. Solving the sovereignty puzzle in E.U. Studies. In: Journal of International Affairs 482, S. 389-431.

Weinhauer, Klaus (2013): Kriminalität in europäischen Hochhaussiedlungen: Vergleichende und transnationale Perspektiven. In: Informationen zur modernen Stadtgeschichte, Nr. 1, S. 35-47. 